\title{
Use of medical titles by non-doctors can mislead patients
}

We failed to send a proof to the authors for this Personal View (BMJ 2011;343:d4241, doi:10.1136/bmj.d4241) and did not inform the authors of our policy that such articles shoud have only one author or of the changes to authorship that we made. Tahwinder Upile, who was originally acknowledged at the end of the article, meets the criteria for authorship in addition to Waseem Jerjes, and both should be listed as authors. The version on bmj.com has been corrected to reflect this.

Cite this as: $B M J 2011 ; 343: \mathrm{d} 4865$ 